\title{
Análise comparativa de características tomográficas de nódulos e massas pulmonares benignas e malignas
}

\section{Comparative analysis of tomographic features of benign and malignant nodules and lung masses}

\author{
Kelser de Souza Kock ${ }^{1,2}$, Maria Fernanda Nicolau³, Cicero Inacio da Silva ${ }^{4}$
}

1. Docente da Universidade do Sul de Santa Catarina (UNISUL). 2. Pós-graduando em Ciências Médicas pela Universidade Federal de Santa Catarina (UFSC), SC, Brasil. 3. Discente do Curso de Medicina pela Universidade do Sul de Santa Catarina (Unisul), Tubarão, SC, Brasil. 4. Docente da Universidade Federal de São Paulo (UNIFESP), São Paulo, SP, Brasil.

\begin{abstract}
Resumo
Introdução: a análise quantitativa das características de nódulos e massas pulmonares por meio da tomografia computadorizada pode auxiliar o diagnóstico médico a estratificar o risco de malignidade. Objetivo: comparar características tomográficas de massas e nódulos pulmonares benignos e malignos, identificando a intensidade em escala de Hounsfield e padrões de textura. Método: estudo transversal, quantitativo, descritivo de levantamento. Foram selecionados prontuários de pacientes internados no Hospital Nossa Senhora da Conceição (HNSC) em Tubarão-SC, no ano de 2014 com diagnósticos de neoplasia benigna dos brônquios e do pulmão (NPB) (D143) e neoplasia maligna do lobo superior, do brônquio ou pulmão (NPM) (C34). Resultados: Foi observado que a chance para malignidade esteve relacionada ao sexo masculino OR=9,4 (IC 95\% 1,7-51,1) com p=0,009, área>13,18 cm2 OR=14,6 (IC $95 \%$ 2,6-82,1) com $\mathrm{p}=0,002$, nos parâmetros de textura, a correlação $\leq 0,166550$ OR=10,3 (IC 95\% 2,1-49,2) com p=0,004 e momento inverso da diferença> 0,166550 OR=28,0 (IC 95\% 4,2-184,3) com p=0,001. Conclusão: houve diferença entre os grupos NPB e NPM entre os parâmetros clínico-epidemiológicos e entre algumas características de textura analisadas.
\end{abstract}

Palavras-chave: Nódulo Pulmonar Solitário. Neoplasias Pulmonares. Projeto Auxiliado por Computador.

\begin{abstract}
Introduction: the quantitative analysis of the characteristics of nodules and lung masses by computed tomography can help the medical diagnosis to stratify the risk of malignancy. Objective: to compare the characteristics of benign and malignant pulmonary masses and nodules, identifying the intensity of the Hounsfield scale and texture patterns. Method: cross-sectional, quantitative, descriptive survey. A selection of medical records was made, observing patients hospitalized at Hospital Nossa Senhora da Conceição (HNSC) in Tubarão-SC in the year 2014 with diagnosis of benign neoplasms of the bronchi and lungs (BNL) (D143) and malignant neoplasms of the upper lobe, bronchus or lung (MNL) (C34). Results: It was observed that a chance for malignancy was related to male $\mathrm{OR}=9.4(95 \% \mathrm{CI} 1.7-51.1)$ with $\mathrm{p}=0.009$, area $>13.18 \mathrm{~cm} 2 \mathrm{OR}=14.6(95 \% \mathrm{CI} 2.6-82.1)$ with $\mathrm{p}=0.002$, in texture, the correlation $<0.166550$ $\mathrm{OR}=10.3(95 \% \mathrm{CI} 2.1-49.2)$ with $\mathrm{p}=0.004$ and inverse difference time $>0.166550 \mathrm{OR}=28.0(\mathrm{CI} 95 \% 4.2-184.3)$ with $\mathrm{p}=0.001$. Conclusion: there was a difference between the BNL and MNL groups between the clinical-epidemiological criteria and some texture characteristics analyzed.
\end{abstract}

Keywords: Solitary Pulmonary Nodule. Lung Neoplasms. Computer-Aided Design.

\section{INTRODUÇÃO}

Os exames de imagem são importantes técnicas investigativas que, de forma complementar ao raciocínio médico, possibilitam a determinação e o acompanhamento de achados patológicos, auxiliando no diagnóstico e na conduta terapêutica a ser tomada.

Um dos exames de imagem mais comuns é a radiografia de tórax, que possibilita o rastreamento de doenças pulmonares que se destacam como a terceira causa de óbito entre as Doenças Crônicas Não Transmissíveis (DCNT) ${ }^{1}$. O câncer ocupa a segunda posição e, ademais, segundo a OMS, óbitos por câncer foram os mais prevalentes que qualquer outra causa mortis, com um total de 8,2 milhões de casos no mundo. Entre as ocorrências de câncer, o pulmão é o órgão mais afetado, com 1,59 milhão de óbitos² . O Nódulo Pulmonar Solitário (NPS), foco do presente estudo, é uma lesão que possui causas diversas: carcinoma broncopulmonar (44\%), tuberculose (23\%), tumores benignos $(13 \%)$, metástase $(9 \%)$ e abcessos $(5 \%)^{3}$.

A identificação do NPS pelo radiologista e sua diferenciação são cruciais para que a sequência de tratamento e a conduta sejam adequadas. Caracteriza-se o NPS em função de ser uma imagem isolada, esférica, mais densa que o ar e com bordos definidos, não ultrapassando $3 \mathrm{~cm}$ de diâmetro ${ }^{3}$. Para o diagnóstico, levam-se em consideração, principalmente, características morfológicas, o que limita o alcance da tomografia computadorizada (TC), por si só, de diferenciar a benignidade de um NPS de um carcinoma broncopulmonar ${ }^{4}$.

De acordo com Silva ${ }^{3}$, um nódulo é caracterizado como benigno

Correspondência: Kelser de Souza Kock. Rua: José Acácio Moreira, 787, Dehon, CEP: 88.904-900 - Tubarao, SC - Brasil. E-mail: kelserkock@yahoo.com.br Conflito de interesse: Não há conflito de interesse por parte de qualquer um dos autores.

Recebido em: 31 Maio 2017; Revisado em: 22 Out 2017; 21 Dez 2017; Aceito em: 26 Dez 2017 
se possuir as seguintes características: calcificação difusa, central ou em camadas, limites precisos (liso, circunscrito) em uma lesão que sofre modificação em sua forma com a mudança de decúbito, tempo de duplicação de uma lesão menor que sete dias, ausência de crescimento por mais de dois anos. Ademais, é preciso adequar a hipótese diagnóstica para a realidade epidemiológica e fisiológica do paciente, no que diz respeito à idade, ao sexo e à história fisiológico-social.

Em se tratando dos exames de imagem, exames de tomografia computadorizada (TC) de tórax com alta resolução permitem ainda uma melhor acurácia, pois evitam sobreposição de estruturas anatômicas e facilitam a visualização de eventuais anormalidades ${ }^{5,6}$. Ademais, nesse sentido, a manipulação e pósprocessamento dessas imagens digitais por meio de softwares podem incrementar a hipótese diagnóstica fornecendo outras informações para o raciocínio nosológico ${ }^{5}$.

$\mathrm{Na}$ imagem de $\mathrm{TC}$, a radiodensidade tecidual pode ser obtida pela escala de Hounsfield e ainda podem ser realizadas análises de variáveis relacionadas à textura do NPS, que descrevem a homogeneidade, desordem e diferença de intensidade entre os pixels ${ }^{6}$.

Então, nesta perspectiva, podem-se determinar valores de referência com o intuito de auxiliar no diagnóstico clínico diante da visualização de um NPS, objetivando respaldar com mais propriedade a conduta clínica a ser tomada ${ }^{7}$. Desta forma, como objetivo geral, a proposta deste trabalho é comparar características tomográficas de nódulos pulmonares benignos e malignos, identificando a intensidade em escala de Hounsfield e padrões de textura.

\section{METODOLOGIA}

Foi realizado um estudo transversal analítico, descritivo de levantamento. A população foi composta por prontuários de pacientes que foram internados no Hospital Nossa Senhora da Conceição (HNSC) em Tubarão-SC, no ano de 2014. Para a composição da amostra, foram selecionados os prontuários de pacientes admitidos no HNSC sob a classificação do Código Internacional de Doenças 10 (CID-10) nas categorias D143 (Neoplasia benigna dos brônquios e pulmão e categoria) e C34 (Neoplasia maligna do lobo superior, brônquio ou pulmão). Esta estratificação foi auxiliada pela equipe da tecnologia da informação do HNSC. Como critérios de exclusão, não foram avaliados os prontuários incompletos ou com dados errôneos, as reinternações e aqueles com ausência de exame de tomografia computadorizada (TC).

Este estudo foi submetido ao comitê de Ética em Pesquisa da Universidade do Sul de Santa Catarina sob o CAAE: 54467416.6.0000.5369.

As variáveis coletadas via prontuário eletrônico foram: diagnóstico de internação, idade e sexo. Por meio do Picture Archiving and Communication System (PACS), foi extraído o corte da TC de alta resolução no formato DICOM com maior área transversa do NPS, analisando a radiodensidade média na escala de Hounsfield (HU) e os parâmetros de textura obtidos com o software ImageJ, plataforma Windows 8 .

O Image é um software para análise de imagens em pesquisas científicas, o qual é capaz de analisar dados desde imagens de detecção de placas de trânsito até reconstrução de imagens tomográficas e ainda pós-processamento de raios $X$ e ultrassonografia. Por meio de extensões deste programa, pode-se também gerar análises que mostrem informações relativas à textura ${ }^{5}$. Para esta finalidade, foi utilizado o plugin do ImageJ: Gray Level Correlation Matrices - $\mathrm{GLCM}^{9}$, que avaliou o $2^{\circ}$ momento angular, contraste, correlação, momento inverso da diferença e entropia da região de interesse selecionada. Para segmentação da imagem, foi utilizado o método de limiarização. A Figura 1 demonstra os procedimentos para análise da textura, após segmentação e seleção do corte.

Figura 1. Procedimento para análise da textura.

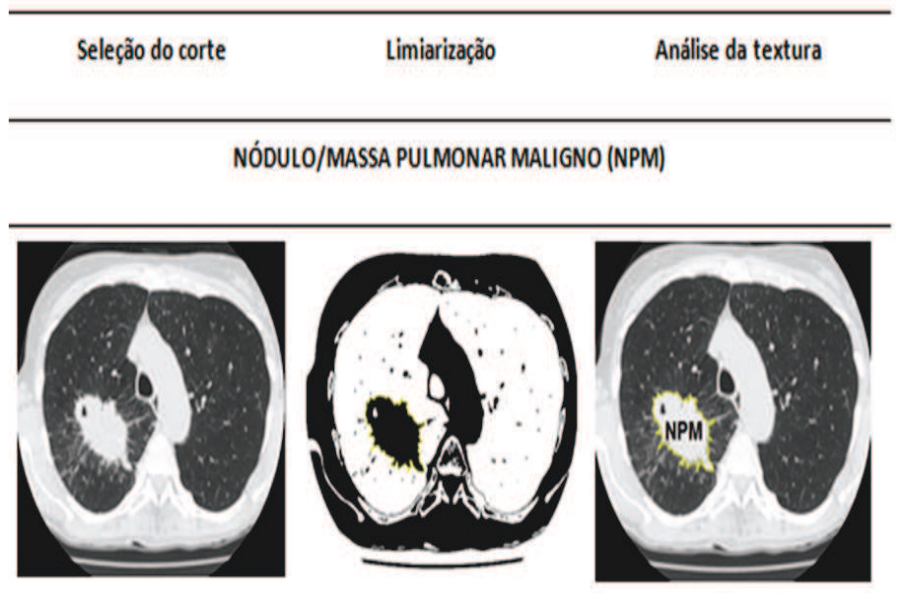

NÓDULO/MASSA PULMONAR BENIGNO (NPB)
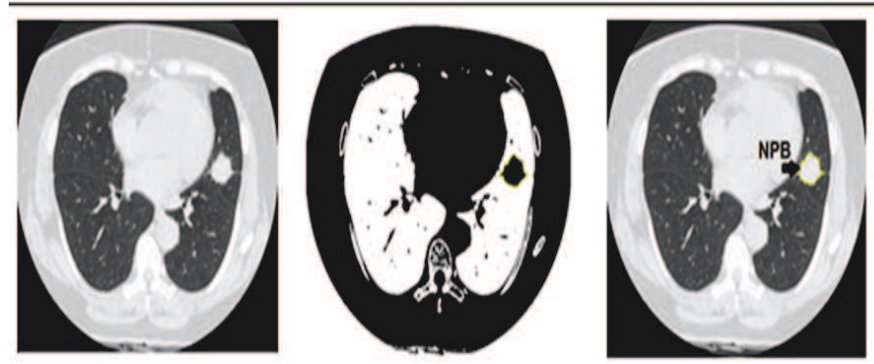

Os dados obtidos foram armazenados no software Excel e analisados estatisticamente pelo software SPSS 20.0. Os resultados foram demonstrados por frequência absoluta e relativa ou por meio de média, desvio padrão absoluto e relativo, dependendo do tipo de variável. Para comparação das diferenças entre os nódulos benigno e maligno, foi utilizado o teste $U$ de Mann-Whitney $(p<0,05)$ para variáveis contínuas e teste exato de Fischer $(p<0,05)$ para as categóricas. A acurácia das variáveis com diferença estatística foi analisada por meio da curva ROC. Em seguida, foi realizada a regressão logística para obtenção do odds ratio para nódulo pulmonar maligno. 


\section{RESULTADOS}

Em primeira análise, foram selecionados 92 prontuários com nódulo pulmonar maligno (NPM) e 15 nódulos pulmonar benigno (NPB). De acordo com os critérios de exclusão, resultaram 34 com NPM e 11 com NPB. A Tabela 1 descreve as características das variáves variáveis analisadas e comparação entre os grupos.

Tabela 1. Características das variáveis e comparação entre os grupos.

\begin{tabular}{|c|c|c|c|c|}
\hline VARIÁVEIS & $\begin{array}{r}\text { AMOSTRA } \\
n=45(100 \%) \\
\text { Média } \pm \text { DP }\end{array}$ & $\begin{array}{r}\text { GRUPO NPM } \\
\mathrm{n}=\mathbf{3 4}(\mathbf{7 5 , 6 \% )} \\
\text { Média } \pm \text { DP }\end{array}$ & $\begin{array}{r}\text { GRUPO NPB } \\
n=11(24,4 \%) \\
\text { Média } \pm \text { DP }\end{array}$ & p \\
\hline \multicolumn{5}{|l|}{ Sexo* } \\
\hline Masculino - $n(\%)$ & $25(55,6)$ & $23(51,1)$ & $2(4,4)$ & \multirow{2}{*}{0,005} \\
\hline Feminino - $\mathrm{n}(\%)$ & $20(44,4)$ & $11(24,2)$ & $9(20,0)$ & \\
\hline Idade (anos) & $63,6 \pm 9,7( \pm 15,3 \%)$ & $64,8 \pm 9,3( \pm 14,4 \%)$ & $59,64 \pm 10,0( \pm 16,8 \%)$ & 0,203 \\
\hline Área $(\mathrm{cm} 2)$ & $28,3 \pm 29,5( \pm 104,2 \%)$ & $34,9 \pm 31,0( \pm 88,8 \%)$ & $7,9 \pm 7,2( \pm 91,1 \%)$ & $<0,001$ \\
\hline Densidade média (HU) & $5,57 \pm 63,3(1136,4 \%)$ & $11,05 \pm 51,6( \pm 467,0 \%)$ & $-11,38 \pm 91,7( \pm 805,8 \%)$ & 0,845 \\
\hline 2 momento angular & $0,0856 \pm 0,214( \pm 250,0 \%)$ & $0,0308 \pm 0,031( \pm 100,6 \%)$ & $0,2550 \pm 0,395( \pm 154,9 \%)$ & 0,457 \\
\hline Contraste & $589799 \pm 449137( \pm 76,2 \%)$ & $597563 \pm 474490( \pm 79,4 \%)$ & $565801 \pm 379189( \pm 67,0 \%)$ & 0,825 \\
\hline Correlação & $0,176 \pm 0,112( \pm 63,6 \%)$ & $0,149 \pm 0,062( \pm 41,6 \%)$ & $0,257 \pm 0,182( \pm 70,8 \%)$ & 0,024 \\
\hline Momento inverso de diferença & $0,262 \pm 0,0964( \pm 36,8 \%)$ & $0,281 \pm 0,0782( \pm 27,8 \%)$ & $0,204 \pm 0,1259( \pm 61,7 \%)$ & 0,032 \\
\hline Entropia & $6580 \pm 722( \pm 11,0 \%)$ & $6588 \pm 690( \pm 10,5 \%)$ & $6557 \pm 849( \pm 12,9 \%)$ & 0,825 \\
\hline
\end{tabular}

As variáveis contínuas com diferença estatística foram analisadas por meio da curva ROC (Figura 2).

Figura 2. Curva ROC das variáveis: área, correlação e momento inverso da diferença.

\begin{tabular}{lcccc}
\hline \multicolumn{1}{c}{ Correlação } & Momento inverso da \\
diferença
\end{tabular}

A Tabela 2 apresenta o odds ratio das variáveis que apresentaram diferença estatística na diferenciação de NPB e NPM.

Tabela 2. Odds ratio bruto das variáveis com diferença estatística.

\begin{tabular}{lrrr}
\hline Variáveis & $\mathbf{n}(\%)$ & OR (IC 95\%) & $\mathbf{P}$ \\
\hline $\begin{array}{l}\text { Sexo } \\
\text { Masculino }\end{array}$ & $25(55,6)$ & $9,4(1,7-51,1)$ & 0,009 \\
$\begin{array}{l}\text { Feminino } \\
\text { Área }\end{array}$ & $20(44,4)$ & 1,0 & \\
$\leq 13,18 \mathrm{~cm} 2$ & $17(37,8)$ & 1,0 & 0,002 \\
$>13,18 \mathrm{~cm} 2$ & $28(62,2)$ & $14,6(2,6-82,1)$ & \\
$\begin{array}{l}\text { Correlação } \\
\leq 0,166550\end{array}$ & $30(66,7)$ & $10,3(2,1-49,2)$ & 0,004 \\
$>0,166550$ & $15(33,3)$ & 1,0 & \\
$\begin{array}{l}\text { Momento inverso } \\
\text { da diferença }\end{array}$ & & & \\
$\leq 0,166550$ & $9(20,0)$ & & \\
$>0,166550$ & $36(80,0)$ & $28,0(4,2-184,3)$ & \\
\hline
\end{tabular}

\section{DISCUSSÃO}

No presente estudo, foi observado que o maior risco relacionado ao NPM e a maior área transversa do nódulo ocorreu com pessoas do sexo masculino. Esses resultados estão de acordo com a literatura, a qual descreve maior malignidade nos homens 
e nos casos em que o tumor ultrapassa três centímetros de diâmetro, sendo, assim, denominado massa em vez de nódulo ${ }^{10}$.

Com relação aos parâmetros de textura, apenas a correlação e o momento inverso da diferença foram associados à malignidade. $\mathrm{Na}$ perspectiva das texturas de $\mathrm{Haralick}^{6}$, a correlação é um parâmetro que indica o quanto um pixel se relaciona com o pixel vizinho, ou seja, demonstra dependência linear de tons de cinza da imagem. Já o momento inverso da diferença descreve pequenas variações e nível de cinza na diagonal principal. Estes parâmetros indicam que, nos casos de NPM avaliados, houve uma maior variação na intensidade de tons de cinza, enquanto, nos NPB, esta variação foi menor.

No entanto, o segundo momento angular, que fornece a medida da homogeneidade local dos níveis de cinza em uma imagem, o contraste, que reflete a quantidade de variação local de níveis de cinza em uma imagem e a entropia, que descreve a desordem da imagem, assumindo valores elevados quando a imagem possui textura não uniforme ${ }^{6}$, não demonstrou diferença estatística entre os NPB e NPM.

Conforme Han et $\mathrm{al}^{11}$, a diferenciação de nódulos pulmonares malignos e benignos é de grande importância clínica, embora a análise das características de textura destas imagens possam ter um papel muito mais importante para predizer malignidade que medidas geométricas. Neste sentido, este estudo comparou três tipos bem conhecidos de recursos de textura bidimensionais (Haralick, Gabor e padrões locais binários) em nódulos pulmonares, indexados no banco de dados público fundado por Lung Image Database Consortium e Image Database Resource Initiative. Como resultados, foi observado que a análise de textura pelo método de Haralick demonstrou maior acurácia (área sob a curva ROC=92,7\%) para diferenciação da malignidade, em comparação com os outros métodos (área sob a curva ROC $=90 \%$ ). Os autores concluem que estas informações podem fornecer um guia para futuras pesquisas na diferenciação de nódulos pulmonares usando imagens de tomografia computadorizada.

Ainda, conforme Dennie et $\mathrm{al}^{12}$, a análise de textura é considerada uma ferramenta de computador que permite a quantificação de padrões de nível de cinza, inter-relações dos pixels e propriedades espectrais de uma imagem, sendo que, por meio desta técnica, há a possibilidade de melhorar os métodos de análise de imagens médicas. Como o câncer de pulmão primário e nódulos granulomatosos têm características muito similares na tomografia computadorizada (TC), os pesquisadores propuseram avaliar a sensibilidade e especificidade por meio da análise de textura e, ainda, comparar a acurácia diagnóstica com a tomografia de emissão de pósitrons (PET). Neste estudo, foram avaliados, retrospectivamente, 55 pacientes com câncer primário de pulmão e nódulos granulomatosos. Os resultados demonstraram que os parâmetros de análise de textura para a TC foram $88 \%$ de sensibilidade e $92 \%$ de especificidade (AUC = $0,90 \pm 0,06, P<0,0001)$. E, na análise da PET, a sensibilidade para a detecção de câncer de pulmão foi de 79,2\% (IC: 57,8-92,9\%), a especificidade foi de $38,5 \%$ (IC: $13,9-68,4 \%$ ) e precisão foi de $64,8 \%$. Como conclusão, os autores ponderam que a análise de textura quantitativa da TC tem o potencial de diferenciar câncer de pulmão primário e lesões granulomatosas.

No presente trabalho, não foi observada a diferença na radiodensidade, mensurada em $\mathrm{HU}$ e na desordem da intensidade de cinza, avaliada pela entropia. Entretanto, no estudo de Suo et $\mathrm{al}^{13}$, foi quantificada a heterogeneidade entre a borda e o núcleo de nódulos pulmonares benignos e malignos por meio de recursos de intensidade e entropia obtidos a partir de análise de textura tomografia computadorizada com contraste. Estes pesquisadores avaliaram 48 nódulos e massas de forma retrospectiva, sendo classificados, histologicamente, em malignidade: inflamação na proporção 24:20. Foi analisado o volume do nódulo e duas regiões distintas: borda e núcleo. Nessas regiões, foram mensurados o nível da escala de cinza em Hounsfield (HU) e entropia, que avalia a heterogeneidade da lesão. Como resultados, foram observadas diferenças significativas nas lesões malignas do que em lesões inflamatórias em relação à intensidade de cinza $(4,71 \pm 5,06 \mathrm{HU}$ versus $-1,53$ $\mathrm{HU} \pm 5,05, \mathrm{p}<0,001)$ e entropia $(0,45 \pm 0,23$ vs $0,18 \pm 0,30, p$ $=0,001)$. Os autores concluem, então, que esta ferramenta é promissora para estimar a probabilidade de malignidade em nódulos e massas pulmonares.

Segundo o autor Miles ${ }^{14}$, os pacientes com câncer de pulmão de não pequenas células (NSCLC) podem apresentar diferentes evoluções clínicas, mesmo quando estão no mesmo estadiamento clínico. Por esse motivo, marcadores prognósticos poderiam auxiliar na estratificação do tratamento para esses pacientes. Neste sentido, informações quantitativas sobre a heterogeneidade do tumor (distribuição de valores de pixel dentro do tumor), como a análise de textura na tomografia computadorizada, podem fornecer informações de prognóstico para pacientes com NSCLC.

A principal limitação deste estudo foi a não confirmação do diagnóstico de internação com o diagnóstico anatomopatológico. Possivelmente, alguns casos de malignidade ou benignidade podem ter sido alocados de forma não adequada nos grupos NPB e NPM. De qualquer forma, pondera-se que o uso de imagens analisadas quantitativamente pode expandir o papel existente do especialista em imagem na avaliação de tumores pulmonares.

\section{CONCLUSÕES}

De acordo com os resultados obtidos, conclui-se que houve diferença entre os grupos NPB e NPM comparando os parâmetros clínico-epidemiológicos e entre algumas características de textura analisadas. Apesar do possível viés de seleção, a ferramenta de análise de textura é uma técnica viável e capaz de discriminar com razoável acurácia tumores pulmonares malignos. 


\section{REFERÊNCIAS}

1. Malta DC, Moura L, prado RR, Escalante JC, Schmidt MI, Ducan BB. Mortalidade por doenças crônicas não transmissíveis no Brasil e suas regiões, 2000 a 2011. Epidemiol. Serv. Saúde. 2014 Dez; 23(4): 599-608.

2. World Health Organization. Cancer. Fact Sheets [Internet]. 2017 Feb [acesso 2017 mar 10]. 297. Disponível em: http://www.who.int/mediacentre/ factsheets/fs297/en/.

3. Silva A. Algoritmos para diagnostico assistido de nodulos pulmonares solitarios em imagens de tomografia computadorizada. Rio Janeiro: PUC; 2004.

4. Koyama H, Ohno Y, Seki S, Nishio S, Yoshikawa T, Matsumoto S, et al. Value of diffusion-weighted MR imaging using various parameters for assessment and characterization of solitary pulmonary nodules. Eur. J. Radiol. 2015 Mar; 84(3): 509-515. doi: 10.1016/j.ejrad.2014.11.024.

5. Abràmofff MD, Magalhães PJ, Ram SJ. Image processing with ImageJ. Biophotonics international. 2004 Jul; 11 (7): 36-42.

6. Haralick RM, Shanmugan K, Dinstein I. Textural features for image classification. IEEE. 1973 Nov; SMC-3(6): 610-62. doi: 10.1109/TSMC.1973.4309314.

7. Castellano G, Bonilha L, Li LM, Cendes F. Texture analysis of medical images. Clin Radiol. 2004 Dec;59(12):1061-9. doi: 10.1016/j.crad.2004.07.008.

8. N. I. of Health, "Download." [Online]. Available: http://rsb.info.nih.gov/ij/ download.html.

9. Cabrera JE, Texture analyzer[Internet]. 2005 [acesso 2017 mar 10]. Disponível em: http://rsb.info.nih.gov/ij/plugins/texture.html.

10. Mosmann MP, Borba MA, Macedo FPN, Liguori AAL, Villarim A Neto, Lima KC. Nódulo pulmonar solitário e 18F-FDG PET/CT. Parte 1: epidemiologia, avaliação morfológica e probabilidade de câncer. Radiol Bras. 2016; 49(1):3542. doi: http://dx.doi.org/10.1590/0100-3984.2014.0012.

11. Han F, Wang H, Zhang G, Han H, Song B, Li L, Moore W, Lu H, Zhao H, Liang Z. Texture feature analysis for computer-aided diagnosis on pulmonary nodules. J Digit Imaging. 2015 Feb;28(1):99-115. doi: 10.1007/s10278-014-9718-8.

12. Dennie C, Thornhill R, Sethi-Virmani V, Souza CA, Bayanati H, Gupta A, et al. Role of quantitative computed tomography texture analysis in the differentiation of primary lung cancer and granulomatous nodules. Quant Imaging Med Surg. 2016 Feb; 6(1):6-15. doi: 10.3978/j.issn.2223-4292.2016.02.01.

13. Suo S, Cheng J, Cao M, Lu Q, Yin Y, Xu J, et al. Assessment of heterogeneity difference between edge and core by using texture analysis: differentiation of malignant from inflammatory pulmonary nodules and masses. Acad Radiol. 2016 Sep; 23(9):1115-22. doi: 10.1016/j.acra.2016.04.009.

14. Miles KA. How to use CT texture analysis for prognostication of non-small cell lung cancer. Cancer Imaging. 2016 Apr 11;16:10. doi: 10.1186/s40644-0160065-5.

\section{Como citar este artigo/How to cite this article:}

Kock KS, Nicolau MF, Silva CI. Análise comparativa de características tomográficas de nódulos e massas pulmonares benignas e malignas . J Health Biol Sci. 2018 Abr-Jun; 6(2):140-144. 